\title{
cuadernos de.
}

UNIÓN EUROPEA PARA LA IDONEIDAD TÉCNICA EN LA CONSTRUCCIÓN junio, 1995

\section{INFORME TÉCNICO DE LA UEAtc PARA LA EVALUACIÓN TÉCNICA DE VENTANAS DE PVC COLOREADAS}

TRADUCCIÓN Y ADAPTACIÓN: J. L. Esteban Sáiz Dr. Ing. Industrial (IETcc-CSIC)

$695-3$ 
El presente documento ha sido elaborado, en común, por los Institutos miembros de la U.E.A.t.c. siguientes:

-El British Board of Agrement (B.B.A.), representante del Reino Unido.

-El Centre Scientifique et Technique du Bâtiment (C.S.T.B.), representante de Francia.

-El Statens Byggeforsknings Institute (S.B.I.), representante de Dinamarca.

-El Forschungsgesellschaft für Wohnen, Bauen und Planen (F.G.W.), representante deAustria.

-El Instituto Eduärdo Torroja de Ciencias de la Construcción (I.E.T.c. c.), representante de España.

-El Instituto Centrale per l'Industrializzazione e la Technologie Edilizia (I.C.I.T.E.), representante de Italia.

-El Laboratorio Nacional de Engenharia Civil (L.N.E.C.), representante de Portugal.

-La Union Belge pour l'Agrément Technique dans la Construction (U.B.A.t.c.), representante de Bélgica.

-El Technical Research Center of Finland (V.T.T), representante de Finlandia.

-El S.I.T.A.C, representante de Suiza.

-El Norwegian Building Research Institute (N.B.I), representante de Noruega.

-El Irish Agrément Board (I.A.B), representante de Irlanda.

-El Epitésügyi Minöségellenörzö Intézet (E.M.I), representante de Hungría.

-El Instytut Techniki Budowlanej (I.T.B), representante de Polonia.

El C.S.T.B. ha sido el ponente.

Conforme a los estatutos de la U.E.A.t.c., cada Instituto miembro, aplica las directrices dadas a continuación, para la concesión en su país, de Documentos de Idoneidad Técnica reconocidos por los otros miembros.

TRADUCCIÓN Y ADAPTACIÓN: J. L. Esteban Sáiz, Dr. Ing. Industrial (IETcc-CSIC) 


\section{PREÁMBULO}

Este informe técnico ha sido preparado por la UEAtc para permitir que los Institutos puedan disponer de las bases de evaluación necesarias para la emisión de un Documento de Idoneidad Técnico Nacional. Con relación a las Guías técnicas de la UEAtc, este informe constituye una base de referencia general, más que una documentación rígida que debe seguir el Instituto acreditador.

En el presente caso, se trata de proponer unos métodos que permitan evaluar las ventanas de PVC coloreadas. El informe técnico indica los principios a seguir para detectar la aptitud de uso con relación a las ventanas de PVC de color blanco que se han realizado, a lo largo del tiempo, como objetivos de trabajo por parte de la UEAtc, de lo cual resulta que numerosas confirmaciones de DIT se han realizado entre Institutos.

De todas formas, teniendo en cuenta la diversidad de métodos de coloreado de los perfiles de PVC y de los métodos de evaluación, es de prever que se establezcan contactos entre el Instituto Emisor y el Instituto Confirmador cuando alguien solicite tal confirmación

\section{ÍNDICE}

\section{CAPÍTULO 1. GENERALIDADES}

1.1 Documentos útiles de referencia

1.2 Principales familias de ventanas de PVC coloreadas

1.3 Designación e identificación

2.2 Sensibilidad a la temperatura

2.3 Aspecto

2.4 Mecanización, fabricación, montaje

2.5 Mantenimiento

2.6 Durabilidad

CAPÍTULO 3. EL PROCEDIMIENTO DE EVALUACIÓN

3.1 Calidad y durabilidad de los perfiles de las ventanas coloreadas de PVC

3.1.1 Generalidades

3.1.2 Durabilidad de los perfiles y del revestimiento

3.1.3 Constancia de la calidad de los perfiles (familia CP) 


\section{CAPÍTULO 1. GENERALIDADES}

\subsection{Documentos útiles de referencia}

-Guía técnica de la UEAtc para la evaluación técnica de las ventanas de PVC (julio 1990)

-Directrices de la UEAtc para la evaluación técnica de ventanas (febrero 1974)

-Proyecto de Norma CEN del TC 33 W 61 T 65, nº 303

-Norma ISO 7724123 . Pinturas y barnices: colorimetría.

\subsection{Principales familias de ventanas de PVC coloreadas}

El anexo 1 proporciona información sobre las diversas técnicas utilizadas.

\subsubsection{Familia CP(Coloured Profiles) (Perfiles Coloreados).}

\section{CPm : coloreado en masa}

La composición vinílica contiene un aditivo pigmentado de color

\section{CPc: coextruidos}

En el momento de la extrusión, las caras elegidas son coloreadas gracias al empleo de un pigmento en una parte (PVC u otra) de la materia a extruir.

\section{CPf: películas}

Después de la extrusión, los perfiles son recubiertos de una película coloreada aplicada la mayor parte de las veces mediante encolado.

\section{CPp: perfiles pintados antes del montaje.}

1.2.2 Familia PW (Painted Windows)(Ventanas Pintadas).

La ventana es pintada después del montaje.

\subsection{Designación e identificación}

El DIT debe indicar claramente, en principio:

-la familia con la que el sistema de coloración de la ventana esté relacionado (por ejemplo, CPc o PW);

-la/las definiciones químicas del eventual revestimiento (por ejemplo, PVC, PMMA,...);

-los elementos eventuales complementarios (adhesivos, por ejemplo);

-las características dimensionales, tales como: espesor, posición del revestimiento.

Además, el Instituto Emisor debe prever un método de identificación que permita verificar:

(c) Consejo Superior de Investigaciones Científicas -la identidad de los colores, valores nominales con tolerancias para los colores, en coordenadas tricromáticas:

- para un mismo lote ;

- para una misma referencia de color ;

-el Instituto emisor apreciará si esas tolerancias son aceptables ;

-la identificación de los materiales (naturaleza química de los revestimientos).

Por último, para las familias ( $\mathrm{PW}, \mathrm{CPp}$ ), se debe indicar con precisión el sistema de renovación y su periodicidad.

\section{CAPÍTULO 2. LOS PROBLEMAS DE PUESTA EN OBRA CON RESPECTO A LAS REGLAS DE EVALUACIÓN}

Los problemas de puesta en obra con relación a las reglas de evaluación

\subsection{Sensibilidad a los choques (fragilidad)}

La resistencia al choque de un perfil es un fenómeno muy complejo que depende de numerosos factores: calidad del polímero, calidad de la extrusión, forma geométrica de los perfiles. Los materiales actuales de PVC, presentan muy buena resistencia al choque y esta propiedad confiere a los perfiles empleados en la carpintería una resistencia al impacto elevada. El reconocimiento de esta propiedad por los que los utilizan ha contribuido notablemente al éxito de las ventanas de PVC, hasta tal punto que los productores de composiciones vinílicas han desarrollado un argumento de venta, mediante formulaciones que incorporan aditivos poliméricos (tipo $\mathrm{B}$ definido en la Guía UEAtc, $2^{\text {a }}$ Parte, Capítulo 1 apartado 1.3) tales como los copolímeros de injertos, mejorando aún más esta propiedad.

El PVC es un polímero lineal irregular de estructura amorfa; su sensibilidad al choque está igualmente fijada al estado de su superficie y a las tensiones internas inducidas durante el proceso de transformación. La aplicación en la superficie de una capa polimérica dando un aspecto coloreado (lacado o por coextrusión) puede provocar una fragilización notable de los perfiles.

Esta particularidad depende del agarre de la capa del polímero, de su naturaleza, de la rigidez de la película de revestimiento y de su espesor. Bajo el efecto del impacto, se reconoce que la fisuración de la capa de revestimiento induce a la rotura frágil del PVC subyacente, fuertemente adherente, por propagación de la fisura inicial.

El fenómeno de debilitamiento del comportamiento al choque es igualmente revelador en los productos revestidos de película, el comportamiento del adhesivo puede ser determinante en esta fragilización.

http://informesdelaconstruccion.revistas.csic.es 
De hecho, hoy, las resistencias al impacto ("Directrices comunes de la UEAtc para la evaluación técnica de ventanas “ ensayo 1.4.2 del capítulo 3 ) de los perfiles con capa de revestimiento coloreado con las mayores prestaciones, son generalmente inferiores a 10 julios a la temperatura de $-10^{\circ} \mathrm{C}$, mientras que los perfiles de PVC clásicos blancos, presentan una prestaciones de 15 a 20 julios.

\subsection{Sensibilidad a la temperatura}

El color resulta de la absorción de la radiación electromagnética del sol. Un cuerpo que absorbe la luz se calienta más, mientras se mantenga el color.

Esto tiende a hacer que la radiación solar transporte energía en el campo del visible ( $45 \%$ de la energía total del espectro de 300 a $700 \mathrm{~nm}$ ) pero, sobre todo, en el campo del infrarrojo (55\% de la energía total del espectro de 700 a $2.000 \mathrm{~nm})$.

La experiencia demuestra que, en Europa, las temperaturas de la superficie, en verano, pueden alcanzar los 45 a $50{ }^{\circ} \mathrm{C}$ sobre los perfiles de PVC blancos; los mismos perfiles de color marrón o negro pueden alcanzar en las mismas condiciones los $75^{\circ} \mathrm{C}$.

Conviene resaltar que las temperaturas de la superficie no dependen solamente del color (longitud de onda), sino también de la calidad de los pigmentos (pinturas con pigmentos reflectantes) o de los fenómenos de superposición de capas.

El calentamiento superficial del PVC, tiene unas consecuencias no despreciables sobre las características fisico-mecánicas del material. El PVC es un material termoplástico que tiene un punto de reblandecimiento (Punto Vicat) bajo carga, situado en torno a los $80^{\circ} \mathrm{C}$. A partir de los $60^{\circ} \mathrm{C}$, la rigidez del material, caracterizada por su módulo de elasticidad, cae considerablemente con la temperatura. El calentamiento de los perfiles bajo los efectos de radiación es un fenómeno limitado a la superficie de los perfiles, por el hecho de la débil conductividad térmica del material. Localmente, bajo los efectos de esta temperatura, el material se dilata y se reblandece: los esfuerzos longitudinales de los perfiles, inducidos por los procesos de extrusión, se liberan y provocan una contracción de las capas de la superficie exterior, de tal forma que las zonas centrales y las superficies interiores de los perfiles, más frías, están poco afectadas.

El fenómeno resultante, conocido con el nombre de efecto bilaminar, origina en obra una concavidad de los perfiles, generando unas flechas permanentes en las carpinterías.

Estas modificaciones de estructura, que producen deformaciones de los perfiles, pueden conducir a un

(c) Consejo Superior de Investigaciones Científicas

Licencia Creative Commons 3.0 España (by-nc) funcionamiento defectuoso (abertura y cierre difícil o imposible), pero son igualmente susceptibles de deteriorar las prestaciones de estanquidad al agua o al aire, pues están ligados a la estanquidad de las juntas.

Las técnicas que se han desarrollado para luchar contra los efectos producidos por los aumentos de elevaciones de temperatura en las ventanas de color, son clásicas: se trata de rigidizar al máximo los perfiles, con la instalación sistemática de perfiles de acero, de fuerte inercia, por el interior de los perfiles de PVC, antes del montaje de los marcos y montantes.

Ciertas concepciones enfocan la limitación del calentamiento mediante una buena aireación de las precámaras de los perfiles exteriores. Esta técnica, poco eficaz, permite disminuir algunos grados la temperatura de la superficie sometida a soleamiento.

Desde este punto de vista, la experiencia adquirida a través de los ensayos en las carpinterías de tinte oscuro ha tomado, como conclusión, que era necesario cuidar especialmente el refuerzo de las carpinterías (fuerte inercia, fijación de los refuerzos) para satisfacer los ensayos de ciclos repetidos de temperatura (entre $-10{ }^{\circ} \mathrm{C}$ y $75^{\circ} \mathrm{C}$ ) ${ }^{(1)}$ y obtener unas deformaciones límites, que no alteren excesivamente las prestaciones de estanquidad al aire.

En fin, conviene tener en cuenta el efecto del calentamiento en los problemas de dilataciones diferenciales, de una parte, con la obra; de otra, con los vidrios.

\subsection{Aspecto}

El coloreado del PVC, cualquiera que sea la técnica empleada, nos lleva al problema de la permanencia del aspecto, con el tiempo, del revestimiento coloreado y de su mantenimiento. Hoy en día, el conocimiento del comportamiento de los perfiles de PVC blancos nos sirven de referencia: el envejecimiento multicapa es un fenómeno de superficie bien conocido y que implica la eliminación de la capa de PVC degradada y que provoca un blanqueamiento lento por la presencia de los agentes opacos $\left(\mathrm{TiO}_{2}\right)$, conservando el material un aspecto idéntico, conduciendo a la consideración de "sin mantenimiento". Esto no resulta igual para las capas coloreadas, que son susceptibles de tener un comportamiento diferente.

Además, el porcentaje de pigmentos (estabilidad de pigmentación), la acción de los estabilizantes sobre ellos, los efectos de cambios suaves de color en los lugares donde hayan estado sometidos a solicitaciones mecánicas (especialmente en las uniones), son otros elementos que juegan un papel importante.

\footnotetext{
${ }^{(1)}$ Esta derogación podrá ser producida a una temperatưra inferiór para los climas moderados del apartado 2.12 (Capitulo 2 de la Guia de la UEAtc para ventanas de PVC) $\mathrm{El}$ argumento deberá ser muy explícito en este tema.
} http://informesdelaconstruccion.revistas.csic.es 
La cohesión del revestimiento eventual, es otro aspecto también a examinar, ya que ello tendrá una incidencia sobre el desprendimiento o la eventual formación de ampollas.

\subsection{Mecanización, fabricación, montaje}

Otro problema de la resistencia al choque, existe en los riesgos suplementarios, debidos a la fragilidad relativa de los perfiles coloreados y que necesitan ser fabricados con ciertas precauciones para la construcción de las ventanas. En este caso, las operaciones de flujo, fresado y pulimento deben ser realizadas con unos utillajes y unas velocidades adecuadas para evitar el desprendimiento y las roturas frágiles.

Es conveniente, sin embargo, tener en cuenta otros dos aspectos suplementarios:

- la soldabilidad de los perfiles coloreados,

- el porcentaje de uniones mecánicas,

que deben ser estudiados, especialmente, en el caso de ventanas coloreadas (calentamiento-relajación).

\subsection{Mantenimiento}

La consideración de débil mantenimiento de las carpinterías de PVC blanco se está investigando también para las ventanas coloreadas. No obstante, el problema debe contemplarse de varias formas:

a) reparación de los desperfectos producidos en obra (rayaduras, ... );

b) limpieza durante el tiempo de la obra;

c) reparación-mantenimiento;

d) pérdida de color después de varios años para las familias PW y CPd.

El Instituto Emisor evaluará las disposiciones propuestas sobre estas consideraciones por el productor.

\subsection{Durabilidad}

La durabilidad del revestimiento está relacionada, de modo esencial, en lo que respecta al problema del aspecto. Ello conlleva, sin embargo, para los perfiles $\mathrm{CPm}$ y CPc y para el material de base de los otros perfiles, que se plantee el problema de interacción del color sobre la durabilidad del PVC.

Además, es conveniente asegurarse de la incidencia del color sobre la durabilidad de las uniones y de la ventana con los productos próximos de la ventana (capa de superficie y material de PVC, masilla, etc.).

Esto resulta muchas veces delicado, siendo conveniente asegurarse de las eventuales incompatibilidades, en particular para la reposición de la pintura (ver tabla l).

Además -y de forma general- el problema de la durabilidad debe ser examinado por:

- el color,

- el material,

- el perfil,

- la ventana.

NOTA: para los sistemas de perfiles pintados o de ventanas pintadas, hay que señalar que cada uno de estos sistemas deben ser objeto de una investigación específica. No es cuestión de evaluar de una forma general la aptitud de una pintura para ser aplicada sobre unos perfiles de PVC.

\section{CAPÍTULO 3. EL PROCEDIMIENTO DE EVALUACIÓN}

\subsection{Calidad y durabilidad de los perfiles de las ventanas coloreadas de PVC}

\subsection{Generalidades}

Los ensayos aplicables a los perfiles de tinte claro son igualmente aplicables a los coloreados:

- resistencia a la tracción y alargamiento en la rotura ;

- módulo de elasticidad en flexión o en tracción;

- resiliencia en tracción o en Texión sobre probetas, entalladas o no.

Salvo para la resiliencia, los resultados son similares a los de PVC blanco (referencia "Guía de la UEAtc sobre ventanas de PVC" (2 $2^{\mathrm{a}}$ Parte, Capitulo $\left.1^{\circ}\right)$.

La resiliencia medida sobre los perfiles de PVC blanco debe estar de acuerdo con las exigencias para este tipo de producto ( 10 julios como referencia "Guía de la UEAtc sobre ventanas de PVC"). Es la misma para los perfiles coloreados en masa; esta resiliencia caracteriza la calidad de la extrusión de los perfiles. Sin embargo se constata que los revestimientos alteran este valor de forma muy sensible. Los ensayos sobre ventanas que permiten decidir la aceptación de los exigencias de resistencia a los choques, son los que se muestran en la tabla 2.

\subsubsection{Durabilidad de los perfiles y del revestimiento}

\subsubsection{Composición vinílica de base}

Cualquiera que sea la familia a la que pertenezca el sistema, es conveniente que la composición vinílica de base sea evaluada de acuerdo con las guías establecidas por la UEAtc ("Guía de la UEAtc sobre ventanas de PVC" $\left(2^{\mathrm{a}}\right.$ Parte, Capítulo $\left.2^{\circ}\right)$. 
TABLA 1

Síntesis de los problemas en obra para las ventanas coloreadas de PVC

\begin{tabular}{|c|c|c|c|c|}
\hline Familia & Color & Perfil & Uniones & Ventana \\
\hline $\mathrm{CPm}$ & $\begin{array}{l}\text { Estabilidad de la } \\
\text { pigmentación. } \\
\text { Acción de los } \\
\text { estabilizadores }\end{array}$ & Calentamiento & $\begin{array}{l}\text { Soldabilidad } \\
\text { Tonalidad de la } \\
\text { soldadura }\end{array}$ & $\begin{array}{l}\text { Fijaciones mecánicas } \\
\text { Deformación } \\
\text { Dilatación }\end{array}$ \\
\hline $\mathbf{C P}$ & $\begin{array}{l}\text { Estabilidad de la } \\
\text { pigmentación }\end{array}$ & $\begin{array}{l}\text { Fragilidad } \\
\text { Calentamiento }\end{array}$ & $\begin{array}{l}\text { Soldabilidad } \\
\text { Tonalidad de la } \\
\text { soldadura }\end{array}$ & $\begin{array}{l}\text { Fijaciones mecánicas } \\
\text { Deformación } \\
\text { Dilatación }\end{array}$ \\
\hline $\mathrm{CPf}$ & $\begin{array}{l}\text { Estabilidad de la } \\
\text { pigmentación. } \\
\text { Adherencia }\end{array}$ & $\begin{array}{l}\text { Fragilidad } \\
\text { Calentamiento } \\
\text { Formación de } \\
\text { ampollas }\end{array}$ & Soldabilidad & $\begin{array}{l}\text { Fijaciones mecánicas } \\
\text { Deformación } \\
\text { Dilatación } \\
\text { Almacenamiento y manipulación }\end{array}$ \\
\hline CPp & $\begin{array}{l}\text { Presentación de } \\
\text { la capa de } \\
\text { pintura }\end{array}$ & $\begin{array}{l}\text { Fragilidad } \\
\text { Calentamiento } \\
\text { Formación de } \\
\text { ampollas }\end{array}$ & $\begin{array}{l}\text { Soldabilidad } \\
\text { Tonalidad de la } \\
\text { soldadura }\end{array}$ & Mantenimiento \\
\hline PW & $\begin{array}{l}\text { Estabilidad de } \\
\text { los colores. } \\
\text { Adherencia de la } \\
\text { pintura. } \\
\text { Constancia de la } \\
\text { calidad }\end{array}$ & $\begin{array}{l}\text { Fragilidad } \\
\text { Calentamiento } \\
\text { Formación de } \\
\text { ampollas }\end{array}$ & Sin objeción & $\begin{array}{l}\text { Fijaciones mecánicas } \\
\text { Deformación } \\
\text { Dilatación } \\
\text { Almacenamiento y manipulación } \\
\text { Mantenimiento }\end{array}$ \\
\hline
\end{tabular}

TABLA 2

Ensayos a realizar

\begin{tabular}{||l|c|c|c||}
\hline \multicolumn{1}{|c|}{ Ensayos } & Familia & Material base & Material + revestimiento \\
\hline $\begin{array}{l}\text { Resist. tracción } \\
\text { Alarg. rotura }\end{array}$ & $\mathrm{CPm}$ & $\mathrm{X}$ & Sin objeción \\
\hline Módulo & $\mathrm{CPc}$ & Imposible de realizar & $\mathrm{X}$ \\
\hline \multirow{3}{*}{ Resiliencia } & $\mathrm{CPf}$ & $\mathrm{X}$ & $\mathrm{X}(1)$ \\
\cline { 2 - 4 } & $\mathrm{CPp}$ y PW & $\mathrm{X}$ & $\mathrm{X}(1)$ \\
\hline \multirow{2}{*}{ (1) Este ensayo permite conocer la incidencia de los revestimientos sobre el comportamiento del material }
\end{tabular}

Para los sistemas CPf, PW y CPp, el ensayo se realiza sobre unos perfiles para colores de base.

Para los sistemas CPm y CPc, el ensayo se realiza sobre unos colores propuestos para el DIT del apartado anterior.

\subsubsection{Comportamiento del color y durabilidad de los perfiles coloreados}

La evaluación se efectúa a partir de una exposición natural de dos años sobre cada tinte propuesto para los CP y sobre el tinte más oscuro ( $\alpha$ máxima) para los $\mathrm{PW}$, en las (c) Consejo Superior de Investigaciones Científicas Licencia Creative Commons 3.0 España (by-nc) condiciones de la tabla 2.1.2 del capítulo $2^{\circ}$ de la $2^{\mathrm{a}}$ Parte de la "Guía de la UEAtc sobre ventanas de PVC".

La evolución es evaluada por la medida de las variaciones de :

- la resistencia a tracción,

- el aspecto,

- la estabilidad de los tintes,

tal como están indicadas en el apartado 2.2 de la misma Guía. 
Estos ensayos son realizados sobre probetas extraídas de los perfiles. Para la familia PW, se asegurará que el espesor del revestimiento aplicado sobre el perfil, esté conforme con el que está previsto aplicar sobre la ventana.

Para los otros tintes propuestos para la familia PW y CPp, debe preverse un ensayo complementario de $8 \mathrm{GJ} / \mathrm{m}^{2}$ bajo degradación de UV (apartado 4.2.1.2 de la "Guía de la UEAtc sobre ventanas de PVC").

NOTA: esta tolerancia aparente, que autoriza el empleo del envejecimiento artificial en lugar del envejecimiento natural, resulta del hecho, por el cual, los sistemas de pintura deben ser renovados periódicamente. Si se presenta el caso contrario, el Instituto deberá pedir que los ensayos sean realizados según el proceso de envejecimiento natural (tabla 3).

\subsubsection{Comportamiento a las solicitaciones mecánicas}

Los ensayos :

- de abrasión: ISO/DIS 77842

- de rayado: ISO 1522

- de cuadrícula: ISO 2409

deben efectuarse de acuerdo con las normas ISO indicadas relativas al comportamiento de pinturas.

Estos ensayos deben ser considerados a las tres temperaturas siguientes: $-10^{\circ} \mathrm{C},+20^{\circ} \mathrm{C}$ y a la temperatura caliente. Son efectuados sobre elementos de carpintería de una ventana -o sobre partes de la misma-.

El Instituto Emisor debe evaluar, según el producto examinado, su sensibilidad a la temperatura.

\subsubsection{Constancia de calidad en los perfiles (familia $C P$ )}

Ver tabla 4.
Además:

- un ensayo que permita caracterizar la constancia de los colores que se ha previsto en cada cambio de suministro; - periódicamente, debe preverse un ensayo de cohesión/ adherencia del revestimiento;

- de forma frecuente, debe ser efectuada una medida del espesor del revestimiento.

\subsection{Calidad y durabilidad de las ventanas coloreadas de PVC}

Los métodos previstos por la "Guía de la UEAtc sobre ventanas de PVC", son enteramente aplicables.

Son completados por los ensayos y disposiciones siguientes:

\subsection{Ensayo de ciclo térmico}

El ensayo $n^{\circ} 3$ "Comportamiento ante las variaciones de temperatura" del capítulo 3 de las Directrices UEAtc de ventanas" se realiza cinco veces de la forma siguiente.

Se mide:

- la permeabilidad al aire después de cada ciclo,

- la evolución de las deformaciones a cada retorno a $20^{\circ} \mathrm{C}$, - la apreciación de la compatibilidad con los extremos de los abombamientos

y se vuelve a anotar al final de los cinco ciclos:

- la degradación eventual del aspecto,

- los malos funcionamientos eventuales en el sistema de maniobra de apertura.

Se recomienda que este ensayo sea realizado sobre la ventana más grande propuesta para su evaluación por el DIT.

TABLA 3

Ensayos a realizar

\begin{tabular}{|c|c|c|c|}
\hline Familias & Composición vinilica de base & Envejecimiento natural & Envejecimiento artificial \\
\hline $\begin{array}{ll}\mathrm{CP} & \\
\mathrm{CPm} \\
\mathrm{CPc} \\
\mathrm{CPf} \\
\end{array}$ & $\begin{array}{l}\text { Sin objeción } \\
\text { Imposible de realizar } \\
\qquad \mathrm{X}\end{array}$ & $\begin{array}{l}\text { Todos los colores } \\
\text { Todos los colores } \\
\text { Todos los colores }\end{array}$ & $=$ \\
\hline PW & $\mathrm{x}$ & El color más fuerte & Otros colores \\
\hline
\end{tabular}


TABLA 4

Constancia de la calidad de los perfiles

\begin{tabular}{||l|l|l|l|l||}
\hline Familias & $\begin{array}{l}\text { Ensayo de constancia } \\
\text { de color }\end{array}$ & \multicolumn{1}{|c|}{$\begin{array}{c}\text { Ensayo de adhesión- } \\
\text { cohesión }\end{array}$} & $\begin{array}{c}\text { Identificación de los } \\
\text { revestimientos }\end{array}$ & \multicolumn{1}{|c||}{$\begin{array}{c}\text { Espesor del } \\
\text { revestimiento }\end{array}$} \\
\hline $\mathrm{CPm}$ & $\begin{array}{l}\text { L.A.B. } \\
\text { espectrocolorimetría }\end{array}$ & Sin objeción & Sin objeción & Sin objeción \\
\hline $\mathrm{CPc}$ & $\begin{array}{l}\text { L.A.B. } \\
\text { espectrocolorimetría }\end{array}$ & ISO.... (tracción) & Espectrofotometría IR & A completar \\
\hline $\mathrm{CPf}$ & $\begin{array}{l}\text { L.A.B. } \\
\text { espectrocolorimetría }\end{array}$ & ISO.... (tracción) & Espectrofotometría IR & A completar \\
\hline $\mathrm{CPp}$ & $\begin{array}{l}\text { L.A.B. } \\
\text { espectrocolorimetría }\end{array}$ & ISO.... (tracción) & $\begin{array}{l}\text { Pintura } \\
\text { Porcentaje de ligante } \\
\text { Extracto en seco } \\
\text { Viscosidad }\end{array}$ & A completar \\
\hline
\end{tabular}

\subsubsection{Ensayo de choque}

El ensayo 1.4.2, del capítulo 3 de las "Directrices de la UEAtc de ventanas", es realizado cinco veces en varios puntos de las caras exteriores de la ventana (o de las partes de las ventanas), situadas en un ambiente $\mathrm{a}-10^{\circ} \mathrm{C}$. No se debe observar degradación.

\subsection{Constancia de la calidad de las ventanas}

El capítulo 5 de la $1^{\text {a }}$ Parte de la "Directrices UEAtc de ventanas" será aplicable totalmente.

Además, para la familia PW, los ensayos de abrasión, rayado, de cuadrícula, espesores (véase el apartado 3.1.2.3 del presente informe), son efectuados sobre muestras tomadas sobre ventanas después de su pintado (tabla 4).

\subsection{Controles externos}

Cuando se trata de las familias $\mathrm{CP}$, la supervisión eventual del autocontrol está basada en los mismos principios que los previstos para los perfiles de ventanas de PVC en blanco.

Esta supervisión se tiene que completar a través de un seguimiento de las unidades de pintura (lacado), para que la operación sea realizada en las dependencias del fabricante de ventanas o en una unidad de aplicación exterior.

\subsection{Varios}

El Instituto debe evaluar la facilidad y el realismo de los dispositivos previstos para la concepción de las ventanas y

(c) Consejo Superior de Investigaciones Científicas Licencia Creative Commons 3.0 España (by-nc) su puesta en obra, principalmente para todo lo que se trate de las disposiciones de refuerzo de los perfiles y las disposiciones constructivas que permitan paliar las dilataciones más grandes de los perfiles de PVC. Esta evaluación está basada en los resultados de los ensayos (especialmente en el apartado 3.2.1), pero también de las experiencias adquiridas sobre las realizaciones ya efectuadas.

Igualmente, para los sistemas de repintado convendría que el Instituto evaluase la facilidad y la fiabilidad.

\section{ANEXO}

\section{PVC coloreado: las diferentes técnicas}

\subsection{Coloración en masa: CPm}

Esta técnica consiste en dispersar unos pigmentos en la masa vinílica; dicha técnica se inició en los años 1970 y se ha desarrollado principalmente en Alemania, hasta tal punto que en los años 1980, el 15\% de las carpinterías se fabricaban en colores oscuros (gris, negro o marrón). -

La utilización de esta técnica decrece actualmente, debido, principalmente, a los problemas de mantenimiento del aspecto, de blanqueamiento, de pérdida de brillo y de clareamiento, que marcan la situación de los tabiques internos de los alveolos de los perfiles, habiéndose producido serios problemas con los colores oscuros.

De forma corriente, en ciertos países, sólo son utilizados en carpinterías colores pastel (gris y beige), que pueden http://informesdelaconstruccion.revistas.csic.es 9 
aceptar una ligera pérdida de brillo, sin consecuencias sobre su aspecto.

Estos productos tienen un excelente comportamiento al envejecimiento.

\subsection{Las láminas encoladas: CPf}

Esta técnica desarrollada por la Sociedad RENOLIT en los años 1970, conducían a unos perfiles de PVC que tenían apariencia de madera.

Este proceso incluye un encolado, por la utilización de un adhesivo, de una lámina de PVC imprimido o estructurado, lámina protegida por otra acrílica sobre el perfil de PVC. Esta lámina acrílica, aplicada a la salida de la extrusión o posteriormente, confiere una buena estabilidad de colores al sistema.

Los principales problemas encontrados han sido debidos a la sensibilidad del sistema por lo que respecta a la manipulación en fábrica y sobre la obra y al riesgo de decoloración en servicio, en particular en el caso de tratarse de colores oscuros.

\subsection{Coextrusión: CPc}

Esta técnica consiste en una extrusión simultánea de un espesor relativamente grueso $(0,5-0,8 \mathrm{~cm})$ de un material acrílico (metacrilato de metilo) y de PVC rígido a la salida de la extrusión.
El revestimiento acrílico conduce a una capa pigmentada lisa, en una larga gama de colores, que es resistente a la imtemperie y que presenta una buena estabilidad de los colores, que tiene una buena resistencia a la abrasión y que limita las elevaciones de temperatura, unidas a la utilización de colores oscuros.

Es necesario tomar ciertas precauciones en fábrica para la confección del producto, pero el comportamiento en obra después de más de diez años no ha presentado problemas de utilización.

\subsection{El lacado : PW}

Los desarrollos recientes en materia de nuevas pinturas (lacas monocapa flexibles con bicomponentes, poliuretanos. acrílicos y poliester) y de imprimaciones específicas al PVC rígido, han conducido a unos productos que presentan las características necesarias de adherencia y de estabilidad a la luz para permitir unos resultados satisfactorios en materia de lacado del PVC.

Unos controles rigurosos en todos los estados del proceso, limpieza y preparación, técnica de aplicación de la pintura, espesor de ésta, polimerización y temperatura de secado adecuadas, son esenciales para conducir a un revestimiento duradero.

Actualmente no existe un sistema de pintura universal para el PVC rígido. Cada sistema de pintura para el PVC rígido debe ser objeto de una investigación especifica, para establecer la compatibilidad 